\title{
DESIGN OF A SNAKE ROBOT BASED ON MODULAR JOINT
}

\author{
YONGJUN SUN*, FENGLEI NI, YUNHU ZHOU, YUANFEI ZHANG, YIWEI LIU, HONG LIU \\ State Key Laboratory of Robotics and System, Harbin Institute of Technology \\ Harbin, Heilongjiang Province, China \\ E-mail: sunyongjun@hit.edu.cn
}

\begin{abstract}
This paper introduces a novel snake robot based on modular joints by harmonic reducer, which have position, torque, acceleration, angular motion, vision and temperature information sensing capabilities. The FPGA chip is used as the joint controller, and the computational powerful computing stick is used as the robot controller in the snake head. The communication between the joint controller and the robot controller is realized through the Point to Point High Speed Serial Communication System (PPSeCo). The prototype was developed and tested for joint output torque, joint desired trajectory and side motion.
\end{abstract}

\section{Introduction}

As early as 1972, Professor Shigeo Hirose of Tokyo Institute of Technology in Japan designed the first snake robot ACM III. The ACM R3 proposed in 2002 adopts the orthogonal axis. The driven wheel is relatively large, which enhances the ability to cross obstacles [1]. ACM R5 uses a bellows for sealing at the joint, which can achieve the underwater gait and turning motion [2], the ACM R8 used a drive wheel design that can span larger obstacles, even twice the height of the robot [3].

Carnegie Mellon University(CMU) has developed a non-wheeled snake robot that can flexibly roll and climb. Early on, they designed a snake robot [4] with a skin that drives the propulsion system, which can pass through small spaces such as barbed wire. Later, the modular snake robot Uncle Sam [5] and SEA Snake [6] were designed to achieve precise torque control, as well as the task of climbing the pole and surveying the pipeline. Sea Snake is equipped with a wide range of sensors for closed-loop control based on position, speed and torque [7]. Due to the high precision of the torque sensors, they have achieved compliant control and joint state estimation based on Kalman filtering [8].

The Norwegian University of Science and Technology (NUST)has developed Anna Konda, Aiko, Wheeko and Kulko. Anna Konda is the earliest snake robot that can perform fire-fighting missions [9]. Professor Y. Pettersen of NUST proposed a non-smooth dynamic model based on the structure of Aiko, which solved the problem of collision modeling of snak robots during motion [10]. Wheeko is a snake robot with a driven wheel. Based on this robot, they built a multi-level closed-loop control model of the integrated position, speed and direction of the wheeled snake robot [11].

Table1. Advantage and disadvantage of wheeled and non-wheeled snake robot

\begin{tabular}{|c|c|c|c|c|}
\hline & & Wheeled snake robot & & Non-wheeled snake robot \\
\hline Advantage & $>$ & $\begin{array}{l}\text { It can better simulate the biological } \\
\text { characteristics of the surface frictional } \\
\text { anisotropy of the biological snake skin, } \\
\text { and realize the gait of the biological } \\
\text { snake. } \\
\text { High speed movement and good } \\
\text { steering ability on smooth roads. }\end{array}$ & $>$ & $\begin{array}{l}\text { The ability to move is more flexible and } \\
\text { adaptable to complex roads. } \\
\text { Can achieve more gait patterns, such as } \\
\text { scrolling and other non-living snake gait. } \\
\text { Can adapt to more mission scenarios, such } \\
\text { as climbing poles. }\end{array}$ \\
\hline Disadvantage & $\vec{\nu}$ & $\begin{array}{l}\text { Poor flexibility } \\
\text { Poor adaptability to uneven road } \\
\text { surfaces. }\end{array}$ & $\vec{\nu}$ & $\begin{array}{l}\text { Difficult to achieve a biological snake gait. } \\
\text { The control is relatively difficult. } \\
\text { Difficult to control in Cartesian space. } \\
\text { Steering performance is poor. }\end{array}$ \\
\hline
\end{tabular}


In this paper, a novel modular non-wheeled snake robot is designed and tested. The main contents are as follows. The second section describes the parameters of the snake robot, the third section $\mathrm{d}$ escribes the mechanical design, the fourth section describes the joint sensor configuration, the fifth section describes the electrical system, and the sixth section describes the control and communication architecture. Finally, a prototype test was carried out.

\section{Snake Robot Specifications}

The snake robot specifications were shown in Table 2.

Table2. Modular snake robot specifications

\begin{tabular}{ll}
\hline Specifications & \multicolumn{1}{c}{ Value } \\
& $\begin{array}{l}\text { Diameter (module joint): } 61 \mathrm{~mm} \\
\text { Length (module joint): } 88.5 \mathrm{~mm}\end{array}$ \\
Dimensions & $\begin{array}{l}\text { Length (full 16 module robot): } 1.416 \mathrm{~m} \\
\text { Diameter (head): } 61 \mathrm{~mm}\end{array}$ \\
& Length (head ): $110 \mathrm{~mm}$ \\
& Module Joint: $187 \mathrm{~g}$ \\
Mass & Full 16 module robot: 2.992kg \\
& Head: 235g \\
Power & Voltage: 28 V \\
Actuators & 28 V Frameless Brushless DC Motor \\
Reducer & Harmonic reducer, Reduction ratio:100:1 \\
Sensors & Motor Angle, Joint Angle, Output Torque \\
Torque & Temperature, 3-axis Accelerometer 3-axis Gyroscope \\
Communication & 3.17Nm \\
\hline
\end{tabular}

\section{Mechanical Design}

Each modular joint has $1 \mathrm{DOF}$, and provides a motion of $\pm 90^{\circ}$. To connect the modules easily, a unified mechanical and electrical interface is applied. The two joints are staggered by 90 degrees, thus simulating the movement of the snake. The snake robot is made up of 16 modules, with head and tail modules.

\subsection{Configuration of Snake Robot}

Inspired by snake, the snake robot was series connected with modular joint. To implement the same function as the ball socket of the snake, the adjacent joint was arranged orthogonally. Each modular joint has independent actuator and sensor system. The configuration of snake robot is shown in Figure 1.

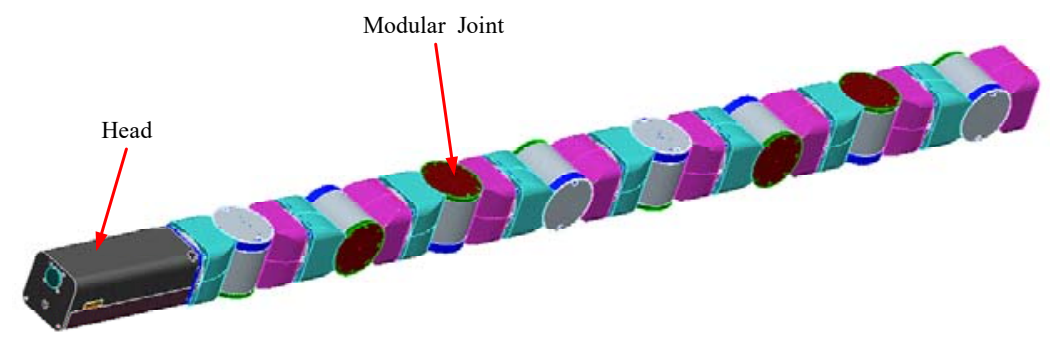

Figure 1. The configuration of snake robot

\subsection{Housing}

The housing of each module is manufactured by 7075 aluminum. And in order to prevent wear and corrosion, it is anodized to natural color. The housing is designed to the special shape which has the following advantages. Not only it can increase the motion range of modular joint, 
but also makes the appearance beautiful. As illustrated in the module cross-section in Figure 2, components are densely assembled inside to minimize volume.

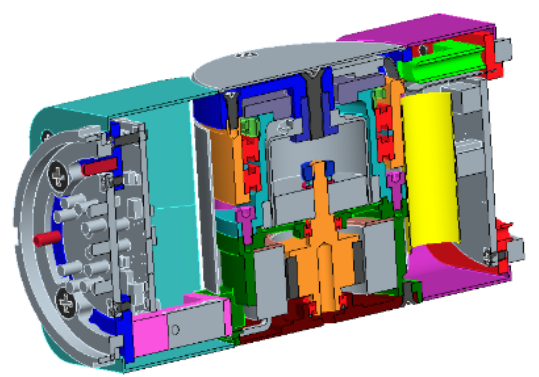

Figure 2. CAD model cross-section of the modular joint

\subsection{Brushless DC Motor}

The snake robot modules are driven by a brushless DC motor which is customized from Micro Motor and Control Institute of HIT. The motor has a rated torque $0.05 \mathrm{Nm}$ with the nominal speed of 2000 RPM.

\subsection{Harmonic Reducer}

The harmonic reducer is a modified HARMONIC DRIVE AG HDUC-8 with a ratio 100:1. Compared to gear reducer, the harmonic reducer does not have the backlash, and has a high precision with small volume. As a result, it has important significance on snake locomotion.

\subsection{Mechanical Interface}

The module of machined grooves at each interface is sealed by O-rings. To ensure the module alignment, two dowel pins disposed at 90 degrees were adopted.

\section{Joint Sensor Configuration}

In order to improve the intelligence level of the joint, which has a rich sensing function, and integrates the joint position sensor, the torque sensor, the motor digital Hall sensor, the temperature sensor, and the sensors such as acceleration and gyroscope, as shown in Table 3.

\begin{tabular}{ccc}
\multicolumn{3}{c}{ Table 3. Joint sensor configuration } \\
\hline Name & Principle & Quantity \\
\hline Joint position sensor & Encoder & 1 \\
Joint torque sensor & Strain gauges & 1 \\
Motor digital Hall sensor & Hall Effect & 3 \\
Temperature Sensor & Temperature sensor chip & 3 \\
Gyroscope & Gyroscope chip & 1 \\
Accelerometer & Accelerometer chip & 1 \\
\hline
\end{tabular}

\subsection{Motor Digital Hall Sensor}

Based on the incremental Hall sensor, it can obtain the position information of the joint angle, which can improve the accuracy and be used to test and decide the rotation direction.

\subsection{Joint Position Sensor}

The joint position sensor is a non-contact magnetic encoder with a large central hole structure. The encoder consists of two parts, a magnetic code disc and a sensitive chip for detection. In order to measure the absolute position information, the magnetic code disc is 
designed based on the dual-code channel method, which uses the cursor to measure the absolute position information.

\subsection{Joint Torque Sensor}

In order to sense the torque of external action and meet the constraints of mass, space and structure, the I-shaped torque sensor is designed. The joint torque sensor is based on the Wheatstone bridge, and the signal output is amplified and filtered for AD conversion. The simulation results are analyzed by ANSYS. The results are shown in Figure 3. It can be seen that the maximum strain of the joint torque sensor is $1007 \mu$ at full scale and the maximum stress is $71.28 \mathrm{MPa}$. The sensitivity and stress meet the requirements of joint measurement torque.

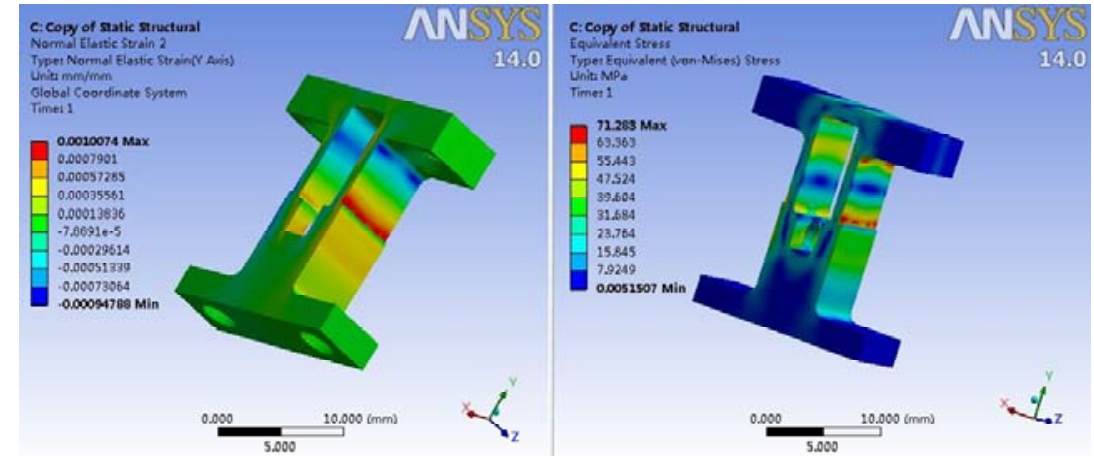

Figure 3. Joint torque sensor finite element analysis cloud

\subsection{Other sensors}

In order to detect information such as posture and acceleration during joint movement, InwenSense's MPU6500 chip is integrated on the joint control board, which integrates a 3-axis accelerometer, a 3-axis gyroscope and a temperature sensor. It can easily acquire the acceleration and posture of the joint. The temperature sensor is used to monitor the temperature of the main control board primarily.

\section{Electrical System}

\subsection{Joint Electrical System}

The electrical system of the joint is integrated inside the joint. The snake robot controller realizes the on-off control of the joint power through the power supply command. The secondary power supply required by the joint internal controller system is converted by the DC/DC power supply system inside the joint, and the joint controller and the robot controller adopt the Point to Point High Speed Serial Communication System (PPSECO) [12]. According to the requirements of joint function and structure, the electrical system inside the joint is divided into four circuit boards, namely, the control board, the joint position acquisition board, the front joint electrical adapter board and the rear joint electrical connection board.

\subsection{Joint Controller}

The joint controller mainly realizes high-speed serial bus PPSECO communication with the robot controller, joint space trajectory planning, joint space position, torque and impedance control and other control algorithms, sensor information acquisition and processing, and joint motor drive control. In order to achieve these functions and improve the integration of the joint 
controller system, an FPGA is used as the joint controller. The functional block diagram of the FPGA is shown in Figure 4.

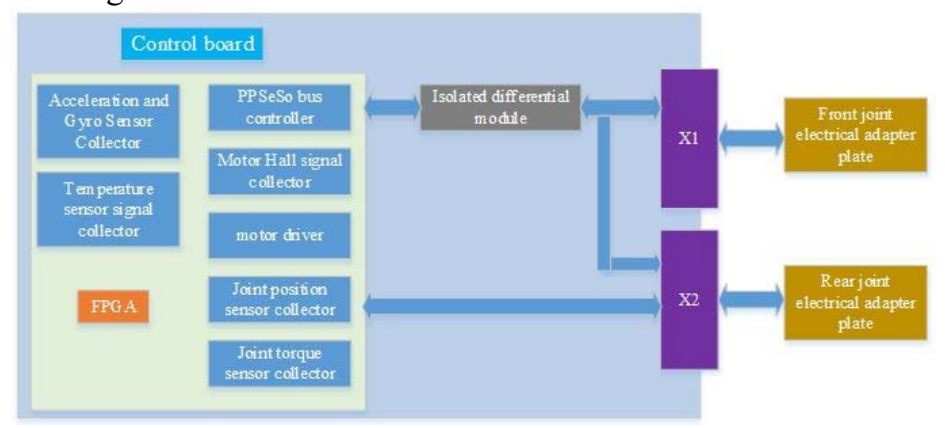

Figure 4. Joint Control FPGA Functional Block Diagram

\subsection{Head Controller}

According to the actual situation, the robot controller is placed on the head of robot. The controller communicates with the joint by PPSECO bus, which is an IP core written based on the VHDL hardware language, so the controller includes an FPGA. On the other hand, the robot controller needs to process the camera vision information and perform gait planning operations, and the FPGA is difficult to handle complex algorithms. Therefore, this paper uses a powerful computer stick with communication interface. The head prototype is shown in Figure 5.

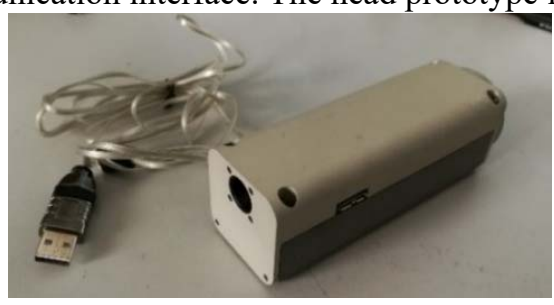

Figure 5 Head prototype

\subsection{Electrical Interface}

Similar to the modular joint mechanical interface, the electrical interface between the joint modules also adopts a modular design concept. The joint module electrical interface includes power and signal lines. Considering the inaccurate installation positioning when installing module joints. Therefore, the electrical interface of joint module adopts the point contact mode of the large pad and the spring contact pin, and the electrical interface is as shown in Figure 6.

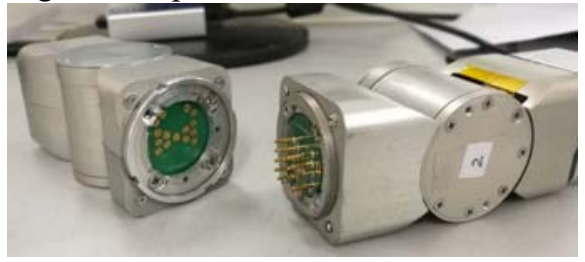

Figure 6 Joint electrical interface design

The snake robot is powered externally. The power connector is installed in the rear space. The tail module mainly implements filtering, overcurrent protection of the external power supply and matching resistors for the joint PPSECO communication.

\section{Control and Communication Architecture}

Fundamentally speaking, the snake robot is a real-time embedded network system. Its main 
functions are to execute motion control commands and feedback sensor information. From a control point of view, the joint module must have low-delay and high-precision motion control information; from a functional perspective, it is desirable to have a flexible communication architecture that is sufficient to support various configurations and feedbacks, and is sufficient to add new type information and control parameters into the module.

\subsection{Communication Protocol}

In order to improve the performance of the snake robot, the joint communication adopts the PPSECO which has been successfully applied to the HIT-DLR dexterous hand, and its communication speed is up to $25 \mathrm{Mbps}$.

\subsection{Control Mode}

The control system is divided into three levels: human-computer interaction level, motion planning control level, and motion-driven control joint-level, as shown in Figure 7.

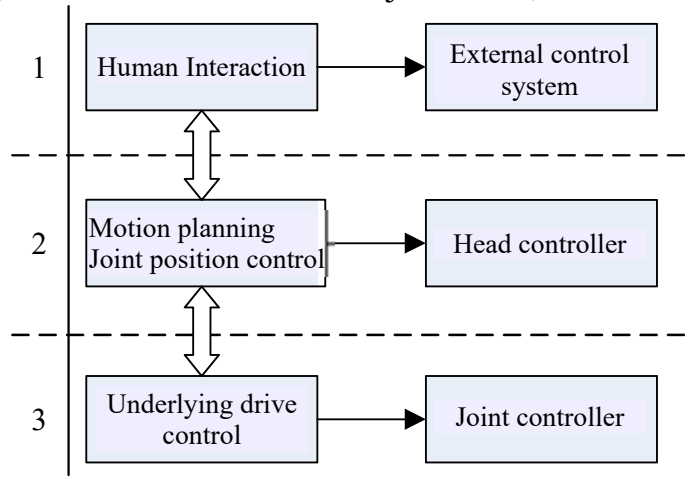

Figure 7. Hierarchical control partition

\section{Prototype Experimental Test}

The prototype was developed and joint output torque, joint desired trajectory and side motion were tested. The prototype of the snake robot developed is shown in Figure 8.

Prototype of snake robot Joint output torque test

Figure 8. Prototype of the snake robot

\subsection{Joint Output Torque}

The joint output torque was tested using a pulley weight system. Test Conditions: the force arm is $56.2 \mathrm{~mm}$; the load mass is $6.75 \mathrm{~kg}$, ignoring the friction between the rope and the pulley, 
therefore, the output torque of the joint is $3.72 \mathrm{Nm}$.

\subsection{Joint Desired Trajectory}

Experimental task: Control the joint angle to make a parabolic swing within $\pm 30^{\circ}$.

According to the trajectory planning, it is necessary to give the expected time and the acceleration. In order to illustrate correctness of the parabolic interpolation algorithm designed in this paper, several sets of controlled experiments were conducted. The experimental conditions are shown in Table 4. During the joint motion, the maximum current $I_{\max }$ and the maximum joint velocity $V_{\max }$ are measured. Joint desired trajectory is shown in Figure 9.

Table 4. Trajectory planning verification experiment

\begin{tabular}{cc}
\hline Experimental condition & Experimental result \\
\hline Experiment $1: t=3 \mathrm{~s}, a=27 \mathrm{deg} / \mathrm{s}^{2}$ & $I_{\max }=0.214 \mathrm{~mA}, V_{\max }=40 \mathrm{deg} / \mathrm{s}$ \\
Experiment $2: t=3 \mathrm{~s}, a=100 \mathrm{deg} / \mathrm{s}^{2}$ & $I_{\max }=0.198 \mathrm{~mA}, V_{\max }=22 \mathrm{deg} / \mathrm{s}$ \\
Experiment $3: t=2 \mathrm{~s}, a=100 \mathrm{deg} / \mathrm{s}^{2}$ & $I_{\max }=0.208 \mathrm{~mA}, V_{\max }=35 \mathrm{deg} / \mathrm{s}$ \\
\hline
\end{tabular}
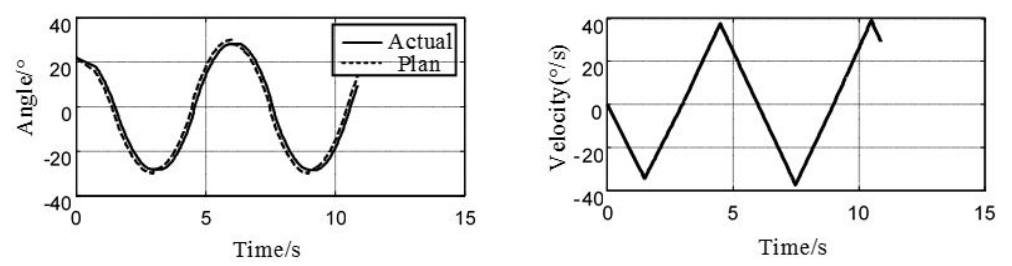

Experiment 1: Joint position and velocity curve
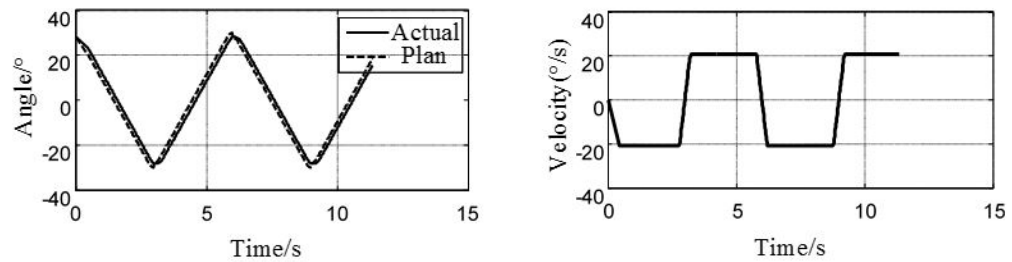

Experiment 2: Joint position and velocity curve
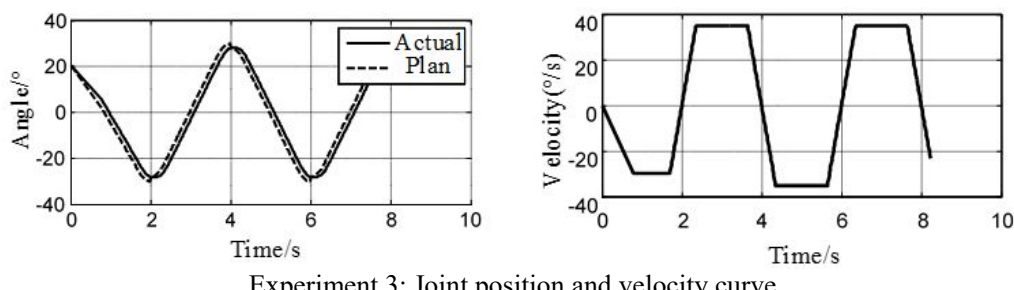

Experiment 3: Joint position and velocity curve

Figure 9. Joint desired trajectory

\subsection{Slide Motion}

The snake robot moves by friction between the joint and the ground. In this paper, take two joints as an example, which can achieve basic two-dimensional motion, such as slide motion.

According to the slide motion condition: the joint motion law is a sinusoidal function, and the adjacent joints are $\pi / 2$ in phase difference. For the joints designed in this paper, the motion law is given in equation (1), and the snake robot can smoothly complete the slide motion, the experimental conditions: $A=25, \square \omega=5.5 \mathrm{rad} / \mathrm{s}$. Figure 10 shows the side shift distance $x$ of the slide motion at different times, the average slide velocity is $7.9 \mathrm{~mm} / \mathrm{s}$.

$$
\left\{\begin{array}{l}
\theta_{1}=A \sin \omega t \\
\theta_{2}=A \cos \omega t
\end{array}\right.
$$



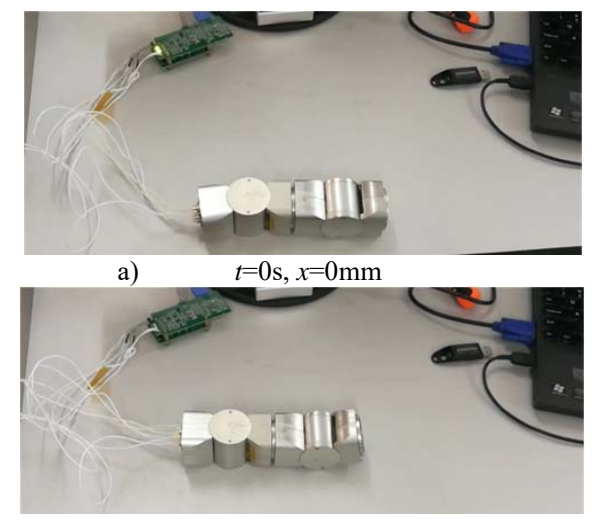

c) $\quad t=10 \mathrm{~s}, x=82 \mathrm{~mm}$

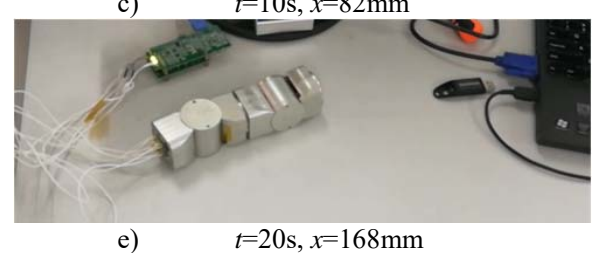

e) $\quad t=20 \mathrm{~s}, x=168 \mathrm{~mm}$

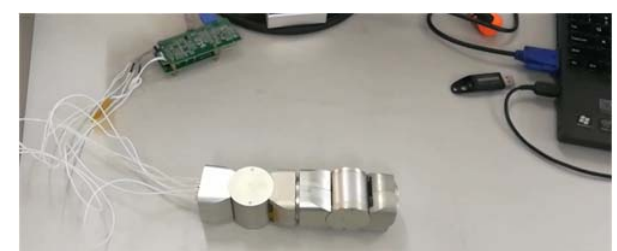

b) $\quad t=5 \mathrm{~s}, x=43 \mathrm{~mm}$

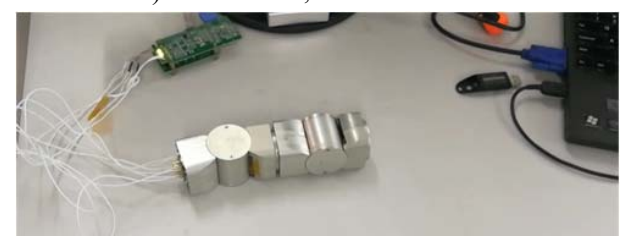

d) $\quad t=15 \mathrm{~s}, x=122 \mathrm{~mm}$

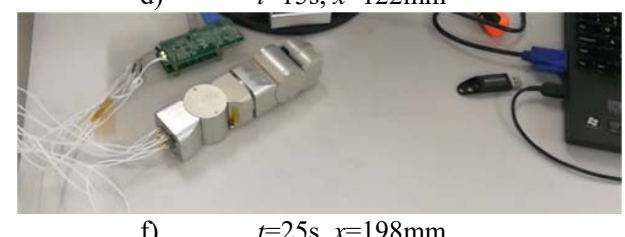

f) $\quad t=25 \mathrm{~s}, x=198 \mathrm{~mm}$

Figure 10. Slide motion

\section{Conclusions}

A novel snake robot based on modular joint was designed, simulated and tested. In the paper, the robot parameters, mechanical design, joint sensor configuration, electrical drive system, command and communication were described in detail. The prototype was developed and tested for joint output torque, joint desired trajectory and side motion.

\section{Acknowledgments}

This work was supported by the National Natural Science Foundation of China (51805107, 51775129, 51521003, 61690210); China Postdoctoral Science Foundation Funded Project (Project No.: 2018M641818); National Key R\&D Program of China (2017YFB1300400), and other Project (SKLRS201720A, 1112881).

\section{References}

1. Mori M and Hirose S. Ieee/rsj International Conference on Intelligent Robots and Systems. IEEE, 829-834, (2002).

2. Yamada H and Chigisaki S. The 36th International Symposium on Robotics, (2005).

3. Komura H, Yamada H, Advanced Robotics, 29(17), 1081-1094,(2015).

4. Anhalt D J, Herron J B. US 7387179 B2[P]. (2008).

5. Liljebäck P and Pettersen K Y, Ø, Robotics \& Autonomous Systems, 60(1), 29-40,(2012).

6. Rollinson D and Bilgen Y, IEEE/RSJ International Conference on Intelligent Robots and Systems, 4630-4636. (2014).

7. Rollinson D and Alwala K V, IEEE/RSJ International Conference on Intelligent Robots and Systems. 1093-1099 (2014).

8. Rollinson D and Choset H. Journal of Field Robotics, 33(3),322-336,(2016).

9. Liljebäck P. Snake Robots: Modelling, Mechatronics, and Control, Springer, (2013).

10. Transeth A A and Leine R I. IEEE Transactions on Robotics, 24(2), 361-376, (2008).

11. Mohammadi A and Rezapour E, IEEE Transactions on Control Systems Technology, 24(3),884-899,(2016).

12. Liu Y and Jin M, Journal of Mechanical Engineering, 45(11), (2009). 